\title{
Immunopurification of adenomatous polyposis coli (APC) proteins
}

\author{
Kerryn L Elliott ${ }^{1,3}$, Bruno Catimel ${ }^{1,4}$, Nicole L Church ${ }^{1,5}$, Janine L Coates ${ }^{1,5}$, Antony W Burgess ${ }^{1,5}$, \\ Meredith J Layton ${ }^{2}$ and Maree C Faux ${ }^{1,5^{*}}$
}

\begin{abstract}
Background: The adenomatous polyposis coli (APC) tumour suppressor gene encodes a 2843 residue (310 kDa) protein. APC is a multifunctional protein involved in the regulation of $\beta$-catenin/Wnt signalling, cytoskeletal dynamics and cell adhesion. APC mutations occur in most colorectal cancers and typically result in truncation of the C-terminal half of the protein.

Results: In order to investigate the biophysical properties of APC, we have generated a set of monoclonal antibodies which enable purification of recombinant forms of APC. Here we describe the characterisation of these anti-APC monoclonal antibodies (APC-NT) that specifically recognise endogenous APC both in solution and in fixed cells. Full-length APC(1-2843) and cancer-associated, truncated APC proteins, APC(1-1638) and APC(1-1311) were produced in Sf9 insect cells.

Conclusions: Recombinant APC proteins were purified using a two-step affinity approach using our APC-NT antibodies. The purification of APC proteins provides the basis for detailed structure/function analyses of full-length, cancer-truncated and endogenous forms of the protein.
\end{abstract}

Keywords: Adenomatous polyposis coli, Colon cancer, Antibody, Protein purification

\section{Background}

The adenomatous polyposis coli (APC) gene is mutated in the germline of individuals with familial adenomatous polyposis (FAP) and in more than $80 \%$ of sporadic colorectal cancers [1-3]. APC encodes a large, multifunctional protein $(2843 \mathrm{aa}, 310 \mathrm{kDa})$ and contains protein-binding domains that have implicated APC in a range of cellular functions. APC is best characterised for its role in the down-regulation of $\beta$-catenin and the Wnt signalling pathway through interactions with $\beta$-catenin and Axin [4-8]. APC also functions in cytoskeletal organisation, cell migration and adhesion $[9,10]$ via interactions with cytoskeletal proteins, such as tubulin, actin, EB1 and discs large protein [11-14].

$A P C$ mutations are generally missense mutations that introduce a premature stop codon, leading to expression

\footnotetext{
* Correspondence: faux@wehi.edu.au

'Ludwig Institute for Cancer Research-Parkville branch, Parkville, VIC 3050, Australia

${ }^{5}$ Current address: The Walter and Eliza Institute of Medical Research, 1G Royal Parade, Parkville, VIC 3052, Australia

Full list of author information is available at the end of the article
}

of truncated APC proteins [1]. The majority of mutations in $A P C$ are confined to a mutation cluster region (MCR) [2] encompassing the $\beta$-catenin and Axin binding sites. Truncation of APC disrupts key binding sites in the C-terminus of the protein, including interactions with the Wnt signalling proteins and both actin and microtubule cytoskeletons. It is now established that APC truncation leads to aberrant regulation of $\beta$-catenin which results in increased transcription of Wnt target genes $[15,16]$.

Despite the importance of APC in colorectal cancer, little is known about the biophysical properties and/or structure of the APC protein or its cancer-truncated forms. Limited structural information on APC has come from studies using small fragments of APC, usually in complex with other proteins. The N-terminus of APC was crystalised as a coiled-coil dimer $[17,18]$, and the 15 and 20 aa repeats were crystalised as fragments with $\beta$-catenin $[19,20]$. However, as these studies used small fragments rather than the full-length APC protein, the structural differences and implications for protein binding between full-length or the truncated APC are not yet known. 
In the present study, we describe the characterisation of new APC monoclonal antibodies and their use in the purification of recombinant forms of APC. APC monoclonal antibodies were generated to the $\mathrm{N}$-terminus of APC, and antibody clones were selected by a combination of ELISA, immunoprecipitation and biosensor analysis. The antibodies were then further characterised by immunoprecipitation and immunofluorescence of endogenous APC. Full length (fl-APC) and truncated APC proteins (APC (1-1638) and APC(1-1311)) were generated using baculoviral-mediated expression in Sf9 cells and purified using a two-step affinity strategy involving immobilised metal affinity chromatography (IMAC) and APC monoclonal antibodies.

\section{Results}

\section{Generation of novel APC monoclonal antibodies}

The N-terminus of APC has been shown to form a coiled-coil structure and dimerise in solution [21]. An $\mathrm{N}$-terminal fragment of APC (residues 1-61) was used as an antigen to raise monoclonal antibodies. This region of APC forms a dimer (not shown) and was therefore thought to mimic the structure of the same region in fulllength APC.

Anti-APC-NT mouse monoclonal antibodies were produced and clones were screened for immunoreactivity to the immunizing antigen by ELISA and surface plasmon resonance (BIAcore) (not shown). Clones that recognised APC-NT were isotyped and analysed for their ability to immunoprecipitate endogenous APC from MDCK epithelial cells (containing wild-type APC) and SW480 colorectal carcinoma cells (containing mutated, truncated APC [22]) (Figure 1B). Both full-length (wild-type) and truncated APC were immunoprecipitated by APC mAb clones 2E7, 6D12, 6G6, 9G11 (all IgG) and 8D9 (IgG2a) (Figure 1B). Therefore APC-NT antibodies can be used to purify endogenous APC proteins, both wild-type and cancer mutated, truncated APC.

In contrast, APC-NT mAbs did not detect APC proteins by Western blot analysis. Cell lysates from MDCK or SW480 cells were separated by SDS-PAGE and immunoblotted with APC-NT or commercial APC antibodies. Only the commercial antibody detected wild-type or truncated APC proteins on immunoblots (not shown). This suggests that APC-NT mAbs can recognise both fulllength and truncated endogenous APC in solution, supporting the prediction that the antigen used had a similar structure to the same region in endogenous, native APC. APC-NT mAbs are unlikely to recognise a linear epitope, as the epitope is not retained upon denaturation of endogenous APC during SDS-PAGE and immunoblotting. Recognition of a conformation-dependent epitope is likely to contribute to the specificity of the APC-NT mAbs for endogenous APC.
These APC-NT mAbs were analysed using BIAcore to determine whether their epitopes overlapped. Purified APC-NT was immobilised onto a BIAcore CM5 chip and the antibodies were injected sequentially over the immobilised peptide. Binding of mAb 6D12 inhibits binding of $6 \mathrm{G} 6$ and $8 \mathrm{D} 9$, and vice versa, suggesting they have at least partially overlapping epitopes, but mAb 2E7 appears to bind independently of the other three antibodies (Figure 1C), suggesting that at least 2 different epitopes on APC-NT are recognised by the APC mAbs.

\section{Generation of APC-encoding baculoviruses}

Baculoviruses encoding fl-APC and truncated APC proteins (APC(1-1638) and APC(1-1311)) containing $\mathrm{N}$-terminal HIS and C-terminal EE [23] affinity tags were generated using the Bac-N-Blue system (Figure 1A). Recombinant APC-encoding baculoviruses were plaquepurified and used for expression of recombinant APC in Sf9 cells. Cells were infected at an MOI of 5 (fl-APC) or an MOI of 1 (truncated APCs) for $72 \mathrm{hr}$ which was found to produce the highest levels of recombinant APC.

The EE-tag was inefficient for purification of the relatively low levels of recombinant APC expressed by the baculovirus. The APC-NT mAbs were therefore tested for their ability to immunoprecipitate recombinant APC proteins, fl-APC, APC(1-1638) or APC(1-1311) from APC baculoviral-infected Sf9 cells (Figure 1D). In APCNT Ab immunoprecipitates, predominant bands were detected at the expected molecular weights of 310, 190 and $150 \mathrm{kDa}$, respectively for fl-APC, APC (1-1638) and APC(1-1311) (Figure 1D, left, middle and right panels, respectively). The APC(1-1638) immunoprecipitated using the APC mAbs 2E7 and 8D9 appeared to have a slightly altered mobility on SDS-PAGE compared to that immunoprecipitated with 6D12, 6G6 and 9D11. However, the concentrations of the antibodies in the immunoprecipitations may differ, thus altering the apparent mobility. Taken together this data shows that each of the 5 APC-NT mAb clones, selected for their ability to recognise endogenous APC in solution, also recognise recombinant full-length and truncated APC expressed in insect cells. All five APC$\mathrm{NT}$ mAbs were used to generate an APC mAb affinity matrix by covalently cross-linking $5 \mathrm{mg}$ of antibody $(1 \mathrm{mg}$ each of 2E7, 6D12, 6G6, 8D9 and 9G11) to $5 \mathrm{ml}$ of Protein G-Sepharose beads.

\section{Characterisation of APC-NT mAbs by immunofluorescence and APC-directed siRNA treatment}

We next assessed whether the APC-NT mAbs recognised endogenous APC protein by immunofluorescence. APC-NT mAb staining in MDCK cells was analysed by confocal microscopy and compared to a commercially available APC antibody (anti-APC-H290). In subconfluent epithelial cells, APC decorates the ends of microtubules at 
A)

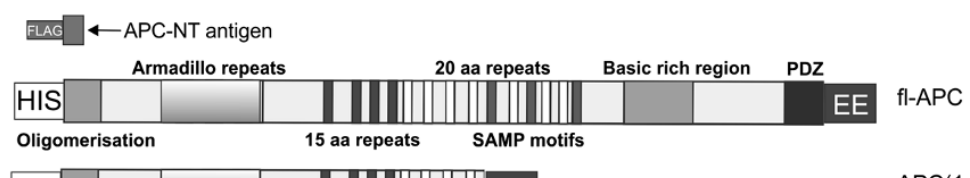

\begin{tabular}{l|l|l|l|l||l|}
\hline HIS & & & & & \\
\hline
\end{tabular}

\section{B) IB:H290 APC}
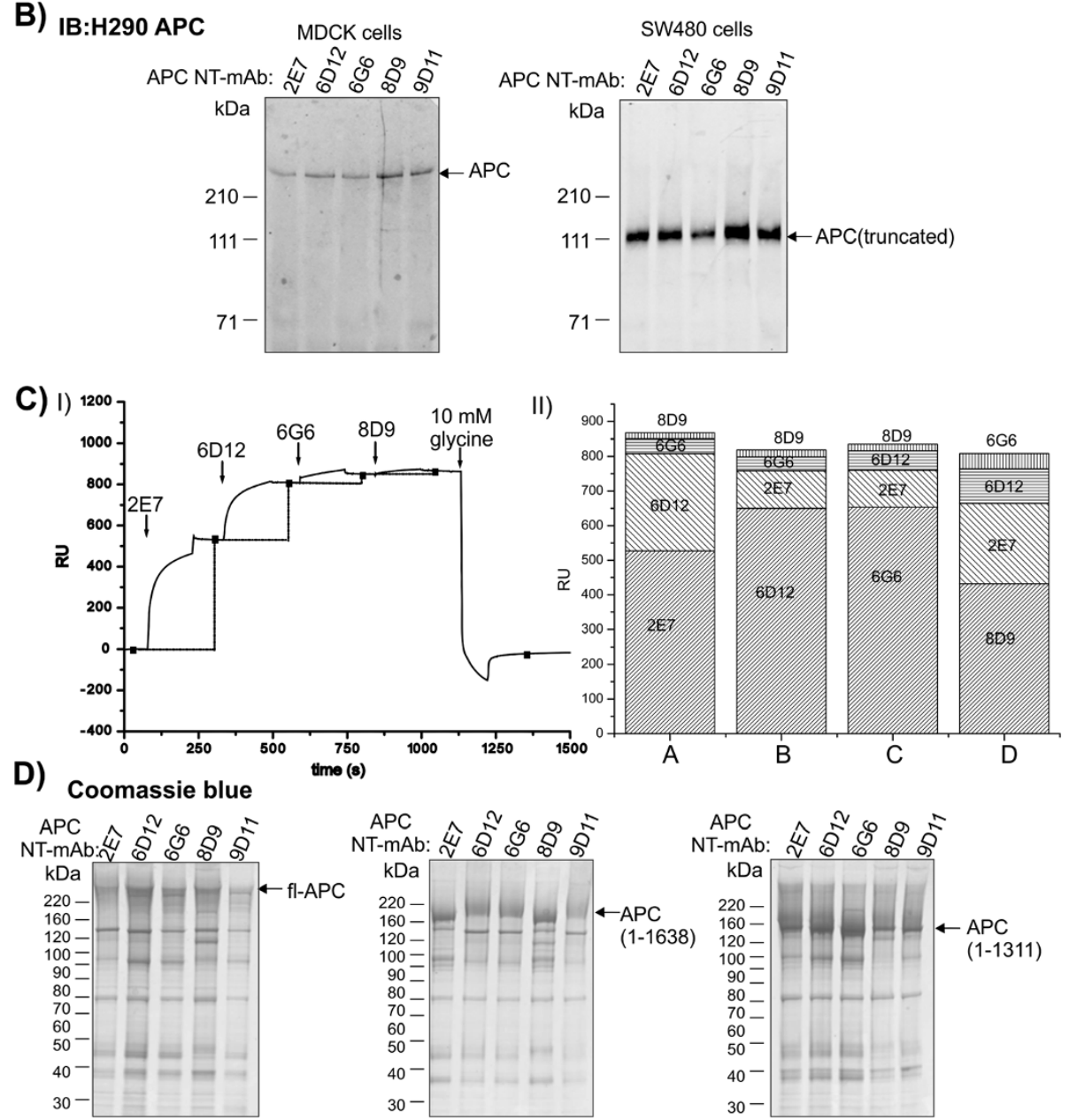

Figure 1 APC-NT mAbs recognize endogenous and recombinant full-length and truncated APC proteins in solution. A) Schematic diagram of structures of recombinant APC proteins. The APC-NT antigen (APC residues 1-61 with an N-terminal FLAG-tag) was expressed and purified from E.coli and used to generate the APC-NT mAbs. Full-length (fl-APC) and truncated recombinant APCs APC(1-1638) and APC(1-1311) were expressed in Sf9 cells with N-terminal HIS and C-terminal EE tags. The protein domains of APC are indicated: Oligomerisation, Armadillo repeats, 15 aa repeats, 20 aa repeats, SAMP motifs, basic rich domain, PDZ domain. Note: the EE-epitope tag was not used for the final affinity purification. B) Immunoprecipitation of endogenous full-length and truncated APC. APC-NT mAb immunoprecipitates from MDCK cells (1 mg protein, left panel) and SW480 colorectal carcinoma cells (1 mg protein, right panel) were immunoblotted (B) with anti-APC H290. C) Biosensor analysis shows overlapping and non-overlapping epitopes for the APC-NT mAbs. I) Representative sensorgram showing sequential injection of APC-NT mAbs and binding to APC-NT antigen. II) Stack graph showing increases in binding (RU) to APC-NT antigen upon sequential injections with different APC-NT mAb clones. Four combinations of APC-NT mAb clones injected sequentially are represented by shaded areas (A-D). Column A corresponds to the sensorgram in I). 2E7 and 8D9 have different epitopes whereas 6D12 and 6G6 share overlapping epitopes. D) Immunoprecipitation of recombinant APC proteins expressed in Sf9 cells. APC-NT mAb $(2 \mu \mathrm{g})$ immunoprecipitates from Sf9 cell lysates $\left(2 \times 10^{7}\right.$ cells) expressing fl-APC (left), APC(1-1638) (middle) or APC(1-1311) (right) were resolved by SDS-PAGE and immunoprecipitated proteins were visualised with Coomassie blue. Note protein immunoprecipitated by 6D12 and $6 \mathrm{G} 6$ migrate slightly differently. The relative positions of the molecular markers are indicated. 
cell protrusions $[24,25]$. The five APC-NT antibody clones each demonstrated the expected staining pattern with concentrated clusters at the ends of microtubule (Figure 2A, representative image shown for mAb 6D12). All APC antibodies recognize APC at microtubule protrusions, however only APC H290 demonstrated nuclear staining and none of the antibodies detected APC at the centrosomes.

We confirmed the specificity of APC-NT antibodies by comparing their immunofluorescent staining pattern in MDCK cells treated with control siRNA with those in which APC levels were depleted using APC-specific siRNAs. Transfection of MDCK cells with siRNAs targeting APC reduced the level of APC by 70\% (Figure 2B). APC staining was still detectable in a proportion of cells, presumably reflecting residual APC protein. However, the typical staining of APC at the ends of microtubules was absent in the majority of APC siRNA transfected cells for each $\mathrm{APC}-\mathrm{NT}$ mAb (Figure 2A, representative image for APC-NT 6D12). Thus each of the APC-NT mAbs specifically recognise endogenous APC localised in clusters at the ends of microtubules.

We next investigated the distribution of APC in SW480 CRC cells with the APC-NT antibodies (Figure 2C, representative image for APC-NT 6G6). In contrast to cells expressing full-length wild-type APC, the truncated APC protein in SW480 cells was not detected at the ends of microtubules but instead was diffusely cytosolic with some concentration of staining in the perinuclear region. This distribution is consistent with the loss of mictrotubule binding in truncated APC and concomitant alterations in cell migration that occur upon $A P C$ mutation.

\section{Purification of recombinant APC proteins}

Having confirmed the specificity of the APC-NT mAbs, a two-step purification scheme for recombinant APC was developed (Figure 3A). Recombinant fl-APC, APC (1-1638) or APC (1-1311) was first purified from clarified Sf9 cell lysates by IMAC. Fractions from this step contained a predominant band at the expected molecular weight as well as a number of minor bands (Figure 3B, left panels). The fractions containing recombinant APC were purified further using APC-NT mAb affinity chromatography. To ensure maximal binding efficiency, the pooled fractions were re-circulated over the APC-NT mAb column three times. Recombinant APC was eluted by disrupting the APC: antibody interaction using low $\mathrm{pH}$. Fractions from this step also contained a predominant band at the expected molecular weight and the levels of minor bands were reduced (Figure 3B, right panels). The fractions containing recombinant APC were pooled for further analysis.

Purified recombinant APC was estimated to be $\geq 80 \%$ pure (Figure $3 \mathrm{C}$ ). The yield of recombinant APC protein was estimated from the intensity of the Coomassie-blue-

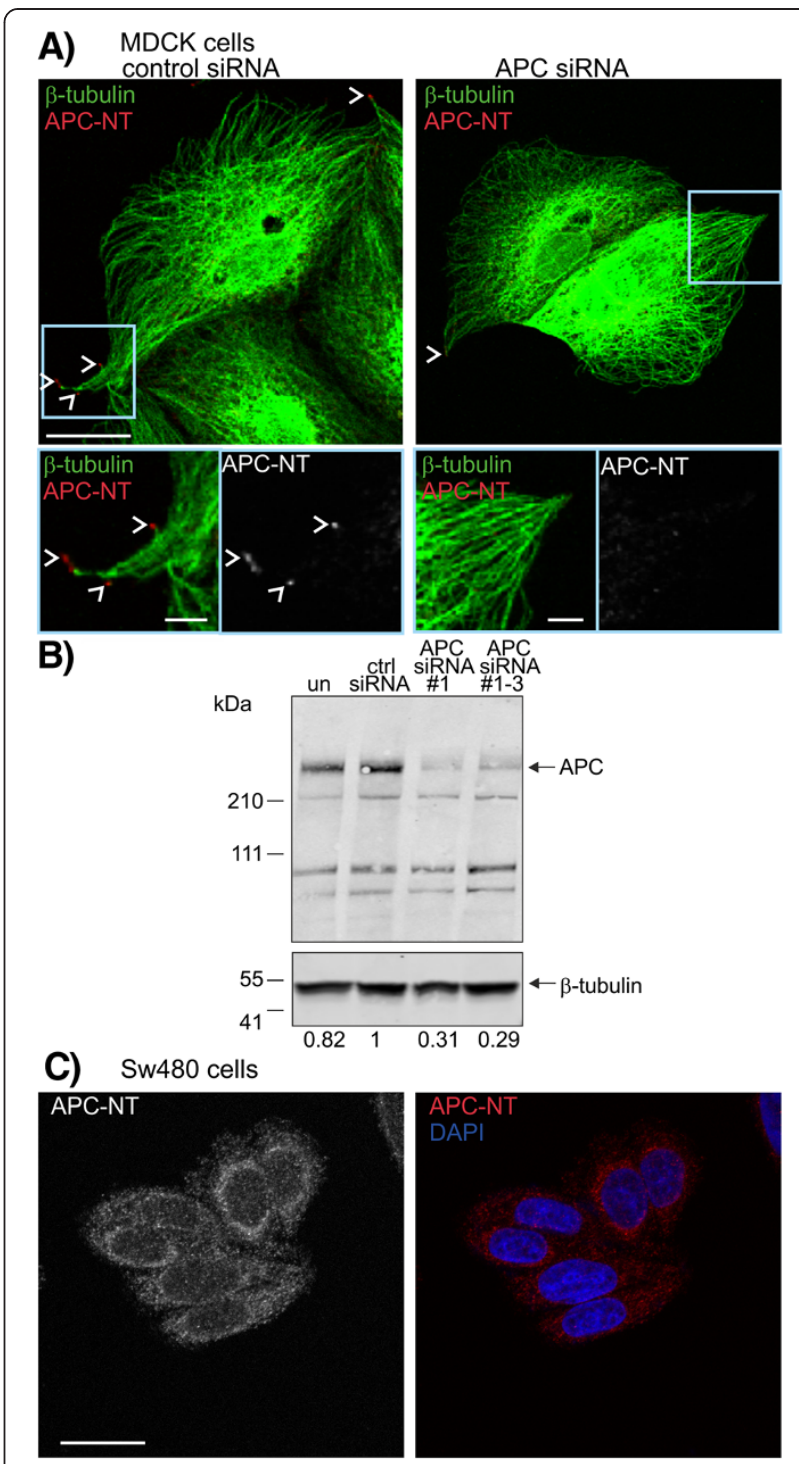

Figure 2 APC-NT mAbs specifically detect APC at the ends of microtubules. A) MDCK cells transfected with control siRNA (top) or APC siRNA (bottom) were immunostained for APC (APC-NT mAb, $6 \mathrm{D} 12$ (red)) and $\beta$-tubulin (green). Distinct clusters of APC at the ends of microtubules (arrowheads) are detected by the APC-NT $\mathrm{mAb}$ in control but not APC depleted cells, scale bar $20 \mu \mathrm{m}$. Enlarged view of insets is shown (right, merged image (left) and single channel grayscale for APC (right)). Shown are single section confocal images, scale bar $5 \mu \mathrm{m}$. B) Depletion of cellular APC by siRNA. APC levels following siRNA transfection were assessed by immunoblot analysis (with H290 Ab from Santa Cruz) in cell lysates. Tubulin was used as a loading control. Numbers below blot are the normalised ratio of APC protein compared to control siRNA. The relative positions of molecular weight markers are indicated. C) SW480 CRC cells were immunostained for APC (APC-NT) and costained with DAPI. APC staining is diffusely cytosolic and is not detected in clusters at the ends of microtubules. Shown are single channel grayscale image for APC (left) and merged image (APC, red and DAPI blue), scale bar $20 \mu \mathrm{m}$. 


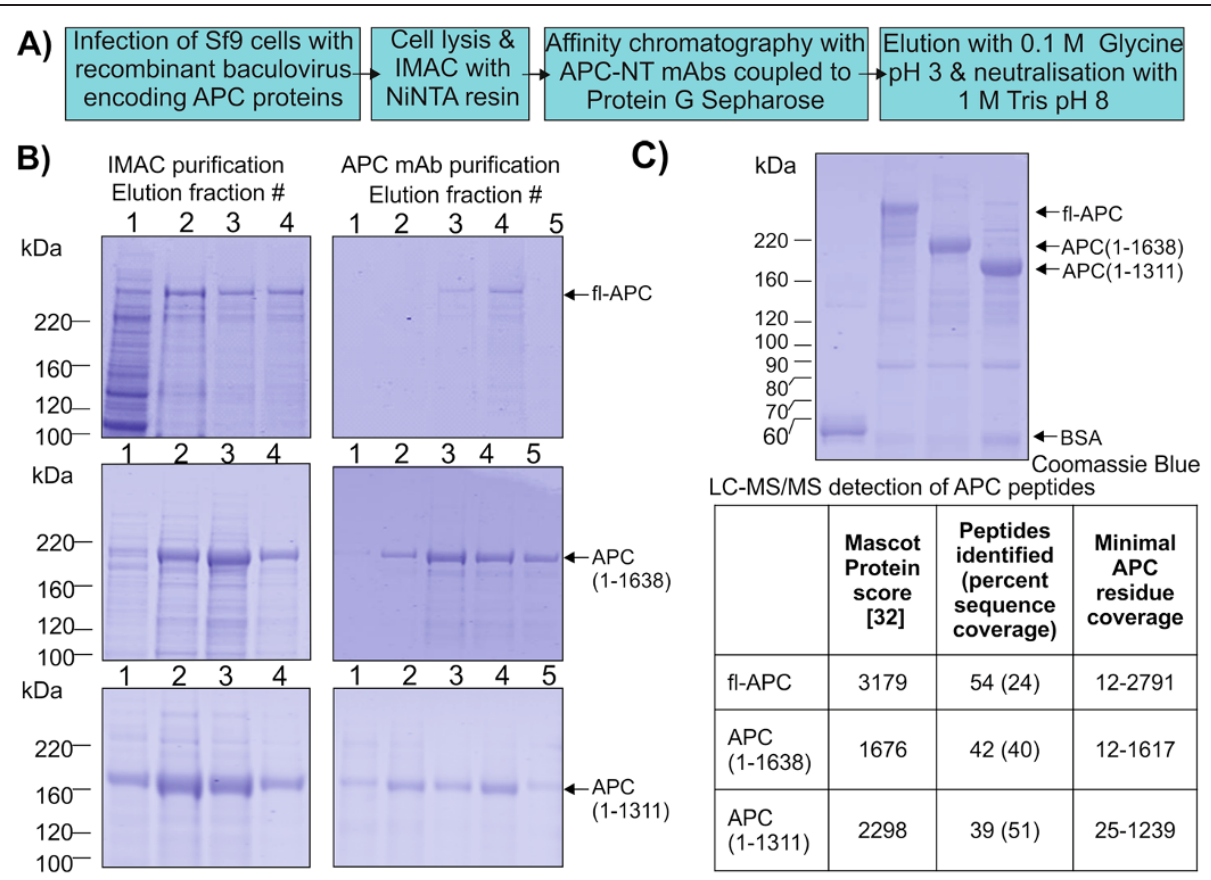

Figure 3 Purification of recombinant APC proteins by a two-step method using IMAC and APC-NT mAb affinity chromatography. A) Schematic diagram depicting the purification scheme used to purify recombinant APC proteins. B) Purification of recombinant APC proteins. Sf9 cells $\left(2 \times 10^{9}\right)$ infected with either fl-APC, APC(1-1638) or APC(1-1311) were and purified using Ni-NTA resin. Proteins were eluted using a stepwise gradient of 20,50, 100 and 250 mM imidazole. 1\% of each fraction was resolved using 4-12\% Bis-Tris SDS-PAGE and stained with Coomassie blue (Left panel, $1=20 \mathrm{mM}, 2=50 \mathrm{mM}, 3=100 \mathrm{mM}, 4=250 \mathrm{mM}$ ). Pooled fractions containing APC were applied to a $5 \mathrm{ml}$ APC-NT mAb column and eluted with $0.1 \mathrm{M}$ Glycine pH 3 in $5 \times 2 \mathrm{ml}$ fractions (right). $1 \%$ of each fraction was resolved as above. The expected sizes of APC proteins are indicated by arrows and relative positions of molecular weight markers in $\mathrm{kDa}$ are indicated. C) Purified recombinant APC proteins. APC proteins were purified using the optimized strategy (A). The amount of APC was estimated by comparison to a band representing $1 \mu \mathrm{g}$ BSA. The expected size of APC proteins are indicated by arrows and relative positions of molecular weight markers are indicated. Purified APC bands were excised and analysed using LC-MS/MS. MASCOT Protein score [26], peptide number, and amino acid coverage are indicated for each protein.

stained band for each recombinant APC relative to the intensity of a band representing $1 \mu \mathrm{g}$ BSA (Figure 3C). The amount of recombinant APC purified from $2 \times 10^{9}$ Sf9 cells was estimated to be 15,250 and $200 \mu \mathrm{g}$ for $\mathrm{fl}-$ APC, APC(1-1638) and APC(1-1311), respectively. The amount of fl-APC purified reflects the low expression levels relative to the expression levels of APC(1-1638) and APC(1-1311).

The identities of the purified recombinant APC proteins were confirmed using LC-MS/MS (Figure 3C). Each excised gel band contained peptides matching human APC (UniProt Accession number P25054). For fl-APC, 54 peptides derived from APC spanning residues 12-2581 (24\% sequence coverage) were identified (Figure $3 C$ ) with a MASCOT protein score of 3179. For APC(1-1638), 42 peptides were identified spanning residues $12-1617$ (40\% sequence coverage, MASCOT protein score 1676) and 39 peptides spanning residues $25-1239$ (51\% sequence coverage, MASCOT protein score 2298) were identified for APC(1-1311). Other proteins identified (other than trypsin and keratin) had MASCOT scores of $<70$, indicating that levels of co-purifying proteins were not significant. The MS data confirmed that the purified recombinant proteins were the expected APC proteins.

\section{Discussion}

The present study demonstrates the generation of APC mAbs that specifically recognise recombinant forms of APC, endogenous APC in solution and the location of APC in fixed cells. Our prediction that using a protein fragment encompassing the $\mathrm{N}$-terminal oligomerisation domain of APC as the immunising antigen would generate antibodies that specifically recognise native, folded APC was correct: each of the APC-NT mAbs recognised native APC. The coiled-coil region was therefore a good antigen for raising monoclonal antibodies that can be used for isolation and detection of endogenous or recombinant, full-length or truncated APC. The APC-NT mAb clones recognized different epitopes and so we used a combination of the five APC-NT mAbs to develop an efficient affinity purification of recombinant APC. 
Each of the APC-NT mAbs detected APC in characteristic clusters at the ends of microtubules in epithelial cells, which is consistent with the previously reported subcellular localisation of APC [24,25,27]. However, in contrast to a commercial APC antibody (H290), none of our APC-NT mAb clones stained the nucleus. There has been some controversy regarding the specificity of commonly used APC antibodies [28]. One APC antibody that was used to show nuclear localisation of endogenous APC was subsequently shown to cross-react with the DNA binding protein Ku80 [29]. Another report used biochemical fractionation and fluorescence microscopy to compare the localisation of APC as detected by a panel of the most commonly used APC antibodies, and found that most of the antibodies tested were unable to specifically detect APC in the nucleus [30]. In SW480 CRC cells that express truncated APC protein, the distribution was diffusely cytoplasmic, consistent with loss of the C-terminal microtubule binding regions of APC. Results from the five APC-NT mAbs reported here support the concept that endogenous APC is predominantly localised at the ends of microtubules in epithelial cells containing wild-type APC, a distribution that is lost upon APC truncation.

\section{Conclusions}

In this study, the APC-NT mAbs were used for the purification of recombinant full-length and truncated APC. Recombinant APC that has been purified by this method can be used for further analysis of APC biochemistry. As these antibodies have been shown to recognise specifically endogenous, native APC, they will also be useful for the study of APC in cells and tissue samples, and for the isolation of APC-containing protein complexes in normal and cancer cells. These reagents will be useful for understanding the structure/function relationships of full-length and truncated APC and the interactions of APC with other cellular proteins.

\section{Methods}

\section{Generation of APC monoclonal antibodies}

APC monoclonal antibodies (APC-NT mAbs) were raised against a fragment of human APC (amino acids 1-61) (accession number P25054) with an N-terminal FLAG epitope tag, produced in E.coli (APC-NT). The fragment was amplified (Forward: 5'-CGCATATGTGCGA TTACAAGGATGACGACGATAAGGGGGGGGCTATG GCTGCAGCTTCGTATGATC-3', Reverse: 5'-GGCTG GATCCTTAAGCCATAGCTTCATC-3') and cloned into $\mathrm{pET} 30 \mathrm{a}+$ for E.coli expression. APC-NT was purified by affinity chromatography on anti-FLAG (M2) Sepharose beads (Sigma) followed by anion exchange (MonoQ HR10/10, GE Healthcare) and reversed-phase HPLC (Discovery C18 $4.6 \mathrm{~mm}$ i.d. $\times 100 \mathrm{~mm}$, Supelco).
Mice were immunised with $3 \times 30 \mu \mathrm{g}$ purified APCNT. Immunisation of mice, fusion of mouse spleens for production of hybridomas and initial screening for APCNT mAbs was carried out by the WEHI Monoclonal Antibody Facility (Bundoora, Australia). Hybridomas were screened further for binding to the APC-NT antigen by ELISA and BIAcore analysis. Five clones (clones 2E7, 6D12, 6G6, 8D9, 9G11) were selected and purified by Protein-A chromatography. Antibodies were isotyped using Isostrip isotyping antibody kit (Roche).

\section{Immunoprecipitation of endogenous APC protein}

MDCK cells [31] were grown in DMEM supplemented with $10 \%$ foetal calf serum and $1 \%$ penicillin/streptomycin. Colorectal carcinoma SW480 cells [22] were grown in RPMI supplemented with $0.01 \mu \mathrm{g} / \mathrm{ml}$ thioglycerol, $0.025 \mathrm{U} / \mathrm{ml}$ insulin, $1 \mu \mathrm{g} / \mathrm{ml}$ hydrocortisone, 10\% FCS and $1 \%$ penicillin/streptomycin. Immunoprecipitations were performed as described [27]. Briefly, clarified cell lysates $(1 \mathrm{mg})$ were incubated with APC-NT mAbs $(2 \mu \mathrm{g})$ for $16 \mathrm{~h}$ at $4^{\circ} \mathrm{C}$ and precipitated with Protein G-Sepharose $(100 \mu \mathrm{l}$, $10 \%$ slurry) (GE Healthcare) for $30 \mathrm{~min}$ at $4^{\circ} \mathrm{C}$. Eluted proteins were analysed by SDS-PAGE (3-8\% Tris Acetate NuPAGE gels) (Novex) and detected using anti-APC H290 antibody (Santa Cruz) followed by anti-rabbit IRDye800 antibody (LiCor) and imaged with the Odyssey infrared imaging system (LiCor).

\section{Biosensor analysis}

All experiments were performed on BIAcore biosensor 2000 (BIAcore). APC-NT protein was exchanged into $10 \mathrm{mM} \mathrm{NaAc}$ pH 4.5 and immobilised on a CM5 chip using NHS-EDC as described previously [32]. Multideterminant binding analysis was performed at a constant flow rate of $20 \mu \mathrm{l} / \mathrm{min}$ for $50 \mu \mathrm{l}$ samples of each APC-NT antibody sequentially, as shown.

\section{Construction and expression of recombinant APC proteins}

Fl-APC was cloned into the pBlueBacHIS2a in three fragments: Firstly, the $5^{\prime}$ end (1-617 bp) was PCR amplified to include a SacI site (Forward: 5 '-GGTGGTGAGCTCATG GCTGCAGCTTCATATG-3', Reverse: 5'-GGAAGAAC AACTAGGTACCTGCCAGG-3') and cloned into pGEM (Invitrogen). Secondly, the mid-region (618-6708 bp) was subcloned by digestion with KpnI and BstBI from pET30amyc-APC [33]. Finally, the $3^{\prime}$ end (6709-8532 bp) was amplified by PCR to include a spacer, EE-tag [34] and Sall site (Forward: 5' -CTCCAACCAACAATCAGC-3', Reverse: 5'-TACCTTGTGACATCTGTTCCCGGGGG GGAGTTCATGCCGATGGAGTGAGTCGACGGATCCATCC-3') and cloned into pGEM. Each fragment was subcloned sequentially (SacI-KpnI, KpnI-BstBI, BstBI-SalI) into pBlueBacHIS2a. 
Truncated APC(1-1638) and APC(1-1311) were cloned in two fragments. Firstly, the $5^{\prime}$ end (1-2156 bp) was amplified to include a $5^{\prime}$ SacI and a $3^{\prime}$ NcoI site (Forward: 5'-GGTGGTGAGCTCATGGCTGCAGCTTCATATG-3', Reverse: 5'-GCACAAAATGATTGCC ATGGACCAC C-3') and cloned into pGEM. Separate 3 ' ends were generated by PCR and cloned into pGEM for APC(1-1638) (2157-4914 bp) (Forward: 5'-GGTGGTCCATGGGA AGTGCTGCAGCTTTAAGGAATCTCAGGCAAATAG GCCTGCG-3', Reverse: 5' -ACACCGGGGGATGATATG CCCGGGGGGAGTTCATGCCGATGGAGTGAG CGA CAGTC-3') and APC(1-1311) (2157-9327 bp) (Forward: 5'-GGTGGTCCATGGGAAGTGCTGCAGCTTTAAGG AATCTCAGGCAAATAGGCCTGCG-3', Reverse: 5'-CC TCCTCAAACAGCTCAACCAAGCCCGGGGGGGAGT TCATGCCGATGGA GTGAGTCGACAGTC-3'). Each fragment was subcloned sequentially (SacI-NcoI, NcoISalI) into pBlueBacHIS2a.

pBlueBacHIS2a-APC cDNAs were co-transfected into Sf9 cells with linearised Bac-N-Blue ${ }^{\mathrm{TM}}$ DNA (Invitrogen). The resulting recombinant baculoviruses were plaque purified and amplified as described [35]. Sf9 $\left(2 \times 10^{7}\right.$ cells $)$ were infected with recombinant baculoviruses at a multiplicity of infection (MOI) between 1 and $5 \mathrm{pfu} / \mathrm{ml}$ to determine the optimum MOI for protein expression.

\section{Immunoprecipitation of recombinant proteins}

Sf9 cells $\left(2 \times 10^{7}\right.$ cells $)$ were infected with the indicated baculoviruses at the optimised MOI. Cells were lysed in Triton X-100 (TX-100) Lysis buffer (Phosphate Buffered Saline (PBS) containing 1\% TX-100 (Sigma) and protease inhibitors (Complete ${ }^{\mathrm{TM}}$ Protease Inhibitor Cocktail (Roche)) $(500 \mu \mathrm{l})$. Lysates were clarified by centrifugation at $16000 \times \mathrm{g}$ for $10 \mathrm{~min}$ at $4^{\circ} \mathrm{C}$ and immunoprecipitated as described above.

\section{siRNA treatment and immunofluorescence}

APC was depleted from MDCK cells using RNA interference as described [27]. MDCK and SW480 cells were lysed for immunoblot analysis or fixed for immunofluorescence as described [27]. Immunofluorescent staining was detected in successive focal planes using an Olympus FV1000 or Nikon C1 confocal microscope equipped with an Olympus $60 \times(\mathrm{NA} 1.35)$ oil immersion lens or Nikon $60 \times$ NA1.35) water immersion lens using standard filter sets and laser lines.

\section{Affinity purification of recombinant APC}

Sf9 cells $\left(2 \times 10^{9}\right.$ cells $)$ were infected with recombinant baculoviruses, harvested after $72 \mathrm{hrs}$, and lysed in TX100 Lysis buffer $+20 \mathrm{mM}$ imidazole. Recombinant APC proteins were affinity purified using IMAC on $1 \mathrm{ml} \mathrm{Ni}$ NTA agarose (QIAgen) equilibrated in $20 \mathrm{mM}$ imidazole, $2 \mathrm{mM} \beta$-mercaptoethanol in PBS, pH 7.5. Unbound proteins were removed with 20-column volumes of the same buffer. Bound APC was eluted with a step gradient of $5 \mathrm{ml} 50 \mathrm{mM}$ imidazole, $2.5 \mathrm{ml} 100 \mathrm{mM}$ imidazole and $2.5 \mathrm{ml} 250 \mathrm{mM}$ imidazole in the same buffer. Fractions were analysed by SDS-PAGE and Coomassie blue staining.

Recombinant APC was further purified by affinity chromatography using a mixture of the 5 APC-NT mAbs. Purified APC-NT mAbs (2E7. 6D12.6D6, 8D9 and 9D11 $5 \mathrm{mg}$ total, $1 \mathrm{mg} /$ clone) were covalently conjugated to Protein G-Sepharose (1 mg Ab/ml) using 50 mM Dimethyl Pimilimidate (Pierce) [36]. Coupling efficiency was determined by SDS-PAGE and Bradford assay, as previously described [36]. The APC-containing fractions from the Ni-NTA step were applied to APC-NT mAb Sepharose equilibrated with PBS containing $2 \mathrm{mM} \beta$-mercaptoethanol and $0.5 \mathrm{mM}$ PMSF. Unbound proteins were removed with 20-column volumes of the same buffer. Bound APC proteins were eluted from the antibody column with two column volumes of $0.1 \mathrm{M}$ Glycine, $\mathrm{pH} 3$ containing $0.5 \mathrm{mM}$ PMSF. Fractions $(2 \mathrm{ml})$ were neutralised immediately with $1 \mathrm{M}$ Tris, pH 8 and analysed by SDS-PAGE as above.

\section{Mass spectrometry}

Coomassie blue-stained bands were excised from SDSPAGE gels and digested in-gel with trypsin [37]. The resulting peptides were analysed by liquid chromatograph coupled to tandem mass spectrometry (LC-MS/MS) (LCQ-DECA, ThermoFinnigan) as described previously [37]. Peak lists were extracted as previously described [37] and searches performed against the LudwigNR protein sequence database (version Q209) (http://www.ludwig.edu. au/archive) using Mascot (version 2.2.04, Matrix Science) with settings of trypsin as the protease, carbamido-cysteine as fixed modification and oxidation of methionine and $\mathrm{N}$-terminal acetylation as variable modifications. All assignments to APC were confirmed by manual inspection of the MS/MS spectra taking into account the Mascot ions score as well as the expectation value. Those peptide spectral matches falling below Mascot's Identity or Homology threshold were accepted only if the annotated spectrum was in accordance with the known rules for peptide fragmentation (Eugene Kapp, personal communication).

\footnotetext{
Abbreviations

APC: Adenomatous polyposis coli; MCR: Mutation cluster region; FAP: Familial adenomatous polyposis (FAP); IMAC: Immobilised metal affinity chromatography; MOI: Multiplicity of infection; mAb: Monoclonal antibody; DME: Dulbecco's modified eagles media; MW: Molecular weight; siRNA: short interfering RNA; TX-100: Triton X-100; EE: Glu or EE epitope tag.
}

Competing interests

The authors declare that they have no conflicts of interest with regard to this manuscript. 


\section{Authors' contributions}

Conceived and designed the experiments: KLE BC MCF MJL AWB. Performed the experiments: KLE NLC JLC MCF. Analyzed the data: KLE MCF MJL. Wrote the paper: KLE AWB MJL MCF. All authors read and approved the final manuscript.

\section{Acknowledgements}

The authors thank Eugene Kapp for mass spectroscopy and Cameron Nowell for microscopy. The work was supported by the National Health and Medical Research Council of Australia Program Grant \#487922, and funds from the Operational Infrastructure Support Program provided by the Victorian Government, Australia. KLE was an Australian Postgraduate Award recipient.

\section{Author details}

'Ludwig Institute for Cancer Research-Parkville branch, Parkville, VIC 3050, Australia. ${ }^{2}$ The University of Melbourne, Parkville, VIC 3050, Australia. ${ }^{3}$ Current address: The University of Gothenburg, Medicinaregatan 9C, Gothenburg, 41390, Sweden. ${ }^{4}$ Current address: Ludwig Institute for Cancer ResearchAustin Branch, Austin Hospital, Level 5, Olivia Newton-John Cancer and Wellness Centre, Studley Road, Heidelberg, VIC 3084, Australia. ${ }^{5}$ Current address: The Walter and Eliza Institute of Medical Research, 1G Royal Parade Parkville, VIC 3052, Australia.

Received: 26 June 2013 Accepted: 21 October 2013 Published: 25 October 2013

\section{References}

1. Fearnhead NS, Wilding JL, Bodmer WF: Genetics of colorectal cancer: hereditary aspects and overview of colorectal tumorigenesis. Br Med Bull 2002, 64:27-43.

2. Powell SM, Zilz N, Beazer-Barclay Y, Bryan TM, Hamilton SR, Thibodeau SN, Vogelstein B, Kinzler KW: APC mutations occur early during colorectal tumorigenesis. Nature 1992, 359(6392):235-237.

3. Smith KJ, Johnson KA, Bryan TM, Hill DE, Markowitz S, Willson JK, Paraskeva C, Petersen GM, Hamilton SR, Vogelstein B, et al: The APC gene product in normal and tumor cells. Proc Natl Acad Sci U S A 1993, 90(7):2846-2850.

4. Hart MJ, De Los Santos R, Albert IN, Rubinfeld B, Polakis P: Downregulation of beta-catenin by human axin and its association with the APC tumor suppressor, beta-catenin and GSK3 beta. Curr Biol 1998, 8(10):573-581.

5. Giles RH, Van Es JH, Clevers H: Caught up in a Wnt storm: Wnt signaling in cancer. Biochim Biophys Acta 2003, 1653(1):1-24

6. Behrens J, Jerchow BA, Wurtele M, Grimm J, Asbrand C, Wirtz R, Kuhl M, Wedlich D, Birchmeier W: Functional interaction of an axin homolog, conductin, with beta-catenin, APC, and GSK3beta. Science 1998, 280(5363):596-599. New York, NY

7. Rubinfeld B, Souza B, Albert I, Muller O, Chamberlain SH, Masiarz FR, Munemitsu S, Polakis P: Association of the APC gene product with B-catenin. Science 1993, 262(5140):1731-1734.

8. Su LK, Vogelstein B, Kinzler KW: Association of the APC tumor suppressor protein with catenins. Science 1993, 262(5140):1734-1737. New York, NY.

9. Nathke I: Cytoskeleton out of the cupboard: colon cancer and cytoskeletal changes induced by loss of APC. Nat Rev 2006, 6(12):967-974.

10. Bienz M, Hamada F: Adenomatous polyposis coli proteins and cell adhesion. Curr Opin Cell Biol 2004, 16(5):528-535.

11. Matsumine A, Ogai A, Senda T, Okumura N, Satoh K, Baeg GH, Kawahara T, Kobayashi S, Okada M, Toyoshima K, et al: Binding of APC to the human homolog of the drosophila discs large tumor suppressor protein. Science 1996, 272(5264):1020-1023. New York, NY.

12. Moseley JB, Bartolini F, Okada K, Wen Y, Gundersen GG, Goode BL: Regulated binding of adenomatous polyposis coli protein to actin. J Biol Chem 2007, 282(17):12661-12668

13. Munemitsu S, Souza B, Muller O, Albert I, Rubinfeld B, Polakis P: The APC gene product associates with microtubules in vivo and promotes their assembly in vitro. Cancer Res 1994, 54(14):3676-3681.

14. Su LK, Burrell M, Hill DE, Gyuris J, Brent R, Wiltshire R, Trent J, Vogelstein B, Kinzler KW: APC binds to the novel protein EB1. Cancer Res 1995, 55(14):2972-2977.

15. Polakis P: Wnt signaling and cancer. Genes Dev 2000, 14(15):1837-1851.

16. Bienz M, Clevers H: Linking colorectal cancer to Wnt signaling. Cell 2000, 103(2):311-320.

17. Day $\mathrm{CL}$, Alber $\mathrm{T}$ : Crystal structure of the amino-terminal coiled-coil domain of the APC tumor suppressor. J Mol Biol 2000, 301(1):147-156.
18. Tickenbrock L, Cramer J, Vetter I, Muller O: Structure of the amino-terminal nuclear export domain of the APC tumor suppressor protein. Ann N Y Acad Sci 2002, 973:109-111.

19. Ha NC, Tonozuka T, Stamos UL, Choi HJ, Weis Wl: Mechanism of phosphorylation-dependent binding of APC to $\beta$-catenin and its role in $\beta$-catenin degradation. Mol Cell 2004, 15(4):511-521.

20. Xing Y, Clements WK, Le Trong I, Hinds TR, Stenkamp R, Kimelman D, Xu W: Crystal structure of a $\beta$-catenin/APC complex reveals a critical role for APC phosphorylation in APC function. Mol Cell 2004, 15(4):523-533.

21. Joslyn G, Richardson DS, White R, Alber T: Dimer formation by an $\mathrm{N}$-terminal coiled coil in the APC protein. Proc Natl Acad Sci U S A 1993, 90(23):11109-11113

22. Leibovitz A, Stinson JC, McCombs WB 3rd, McCoy CE, Mazur KC, Mabry ND: Classification of human colorectal adenocarcinoma cell lines. Cancer Res 1976, 36(12):4562-4569.

23. Porfiri E, Evans T, Chardin P, Hancock JF: Prenylation of Ras proteins is required for efficient hSOS1-promoted guanine nucleotide exchange. J Biol Chem 1994, 269(36):22672-22677.

24. Faux MC, Coates JL, Catimel B, Cody S, Clayton AH, Layton MJ, Burgess AW: Recruitment of adenomatous polyposis coli and $\beta$-catenin to axin-puncta. Oncogene 2008, 27(44):5808-5820.

25. Nathke IS, Adams CL, Polakis P, Sellin JH, Nelson WJ: The adenomatous polyposis coli tumor suppressor protein localizes to plasma membrane sites involved in active cell migration. J Cell Biol 1996, 134(1):165-179.

26. Perkins DN, Pappin DJ, Creasy DM, Cottrell JS: Probability-based protein identification by searching sequence databases using mass spectrometry data. Electrophoresis 1999, 20(18):3551-3567.

27. Faux MC, Coates JL, Kershaw NJ, Layton MJ, Burgess AW: Independent interactions of phosphorylated beta-catenin with E-cadherin at cell-cell contacts and APC at cell protrusions. PLoS One 2010, 5(11):e14127.

28. Davies ML, Roberts GT, Stuart N, Wakeman JA: Analysis of a panel of antibodies to APC reveals consistent activity towards an unidentified protein. Br J Cancer 2007, 97(3):384-390.

29. Roberts GT, Davies ML, Wakeman JA: Interaction between Ku80 protein and a widely used antibody to adenomatous polyposis coli. $\mathrm{Br} J$ Cancer 2003, 88(2):202-205.

30. Brocardo M, Nathke IS, Henderson BR: Redefining the subcellular location and transport of APC: new insights using a panel of antibodies. EMBO Rep 2005, 6(2):184-190.

31. Ridley AJ, Comoglio PM, Hall A: Regulation of scatter factor/hepatocyte growth factor responses by Ras, Rac, and Rho in MDCK cells. Mol Cell Biol 1995, 15(2):1110-1122

32. Nice E, Layton J, Fabri L, Hellman U, Engstrom A, Persson B, Burgess AW: Mapping of the antibody- and receptor-binding domains of granulocyte colony-stimulating factor using an optical biosensor, comparison with enzyme-linked immunosorbent assay competition studies. J Chromatogr 1993, 646(1):159-168

33. Faux MC, Ross JL, Meeker C, Johns T, Ji H, Simpson RJ, Layton MJ, Burgess AW: Restoration of full-length adenomatous polyposis coli (APC) protein in a colon cancer cell line enhances cell adhesion. J Cell Sci 2004, 117(Pt 3):427-439.

34. Porfiri E, Rubinfeld B, Albert I, Hovanes K, Waterman M, Polakis P: Induction of a $\beta$-catenin-LEF-1 complex by wnt-1 and transforming mutants of $\beta$-catenin. Oncogene 1997, 15(23):2833-2839.

35. O'Reilly DR, Miller LK, Luckow VA: Baculovirus Expression Vectors: A Laboratory Manual. New York: Oxford University Press; 1992.

36. Harlow E, Lane DP: Antibodies: A Laboratory Manual. New York: CSHL Press; 1988

37. Mathias RA, Wang B, Ji H, Kapp EA, Moritz RL, Zhu HJ, Simpson RJ: Secretome-based proteomic profiling of Ras-transformed MDCK cells reveals extracellular modulators of epithelial-mesenchymal transition. J Proteome Res 2009 , 8(6):2827-2837.

\section{doi:10.1186/1756-0500-6-429}

Cite this article as: Elliott et al:: Immunopurification of adenomatous polyposis coli (APC) proteins. BMC Research Notes 2013 6:429. 